\title{
étude expérimentale d'une paroi moulée ancrée par quatre nappes de tirants
}

\author{
par \\ H. Josseaume \\ Laboratoire Central des Ponts et Chaussées \\ R. Stenne \\ Entreprise S.I.F.-Bachy
}

\section{Introduction}

Le Laboratoire Central des Ponts et Chaussées, l'entreprise S.I.F.-Bachy et le Laboratoire de Mécanique des Solides de l'Ecole Polytechnique se sont associés pour l'étude expérimentale d'une paroi moulée, réalisée dans le cadre d'une action de recherche concertée de la D.G.R.S.T.* ayant pour objectif une meilleure connaissance du comportement des fouilles à l'air libre retenues par des parois moulées ancrées.

Les critères retenus pour le choix du site de l'expérimentation étaient les suivants :

- la fouille, étayée par la paroi, devait avoir une profondeur d'au moins $15 \mathrm{~m}$,

- les caractéristiques physiques et mécaniques du sol devaient pouvoir être mesurées à partir d'essais classiques faits en laboratoire et en place,

- les caractéristiques hydrauliques devaient être parfaitement définies.

Ce n'est qu'après plus d'une année de recherches qu'un site satisfaisant à ces différents critères a pu être trouvé. 11 s'agit du site de l'immeuble de la S.E.R.E.T.E., édifié rue du Château-des-Rentiers, dans le $13^{\mathrm{e}}$ arrondissement de Paris, dont la construction des niveaux en sous-sol nécessitait l'ouverture d'une fouille d'environ $21 \mathrm{~m}$. de profondeur dans des formations typiques de la région parisienne. La paroi moulée, ancrée par 4 nappes de tirants provisoires, qui devait constituer le soutènement des parois de cette fouille, a été retenue pour l'étude expérimentale envisagée. Des mesures, ayant pour objectif de déterminer aussi précisément que possible les efforts appliqués à cette paroi ainsi que ses déplacements, ont été effectuées sur un de ses panneaux constitutifs pendant toute la durée des travaux intéressant ce panneau, c'est-à-dire entre le 26 mai 1975 et le 29 août 1975 .

\section{Description du site et de la paroi moulée}

La paroi moulée (fig. 1) a été réalisée à partir d'une plateforme établie à $3,30 \mathrm{~m}$. au-dessous de la surface du terrain naturel et débordant l'emprise de l'immeuble. Cette paroi, qui a une épaisseur de $0,60 \mathrm{~m}$, et une hauteur totale de $20,40 \mathrm{~m}$., a été coulée par panneaux alternés (panneaux primaires et secondaires). Elle était ancrée par

* Délégation Générale à la Recherche Scientifique et Technique.
4 nappes de tirants forés du type Bachy, mis en place dans le sol et précontraints au fur et à mesure des terrassements. L'ancrage d'un panneau primaire était assuré par 4 tirants, celui d'un panneau secondaire par 8 tirants.

Les mesures ont été effectuées sur un panneau secondaire, le panneau 34 , situé dans une section rectiligne de l'enceinte (fig. 2). La longueur totale de ce panneau était de $5,32 \mathrm{~m}$. Les caractéristiques des tirants qui assuraient sa stabilité sont indiquées dans le tableau 1.

\begin{tabular}{l|c|c|c}
\hline $\begin{array}{c}\text { Nappe } \\
\text { Type }\end{array}$ & $\begin{array}{c}\text { Longueur } \\
\text { libre } \\
(\mathrm{m})\end{array}$ & $\begin{array}{c}\text { Longueur } \\
\text { de } \\
\text { scellement } \\
(\mathrm{m})\end{array}$ & $\begin{array}{c}\text { Tension } \\
\text { de précontrainte } \\
\text { théorique } \\
\text { (kN) }\end{array}$ \\
\hline 1 TMF & 13 & 9 & 366 \\
2 TMD & 12 & 6 & 611 \\
3 TMD & 6 & 9 & 840 \\
4 TMD & 3 & 9 & 1040 \\
\hline
\end{tabular}

Tableau 1 Caracteristiques des tirants du panneau de mesures

Les formations rencontrées, depuis la surface du terrain naturel, sont les suivantes:

- des alluvions anciennes sablo-graveleuses comportant une forte proportion d'éléments fins,

- le sable de Beauchamp, se présentant ici comme un sable fin très argileux,

- les marnes et caillasses, constituées d'une alternance de marne blanche et de bancs calcaires très fracturés, la marne étant très largement prépondérante,

- le calcaire grossier.

Aucune nappe n'a été décelée dans les sondages de reconnaissance qui ont été poussés jusqu'au toit du calcaire grossier.

Un sondage avec prélèvement d'échantillons intacts, spécialement exécuté dans la perspective d'une étude détaillée du sol au voisinage immédiat du panneau de mesures, a permis de situer les limites des couches dans la zone intéressée par les mesures (fig. 1). Le toit du calcaire grossier non représenté sur cette figure se situe approximativement à la cote 32 N.G.F. 

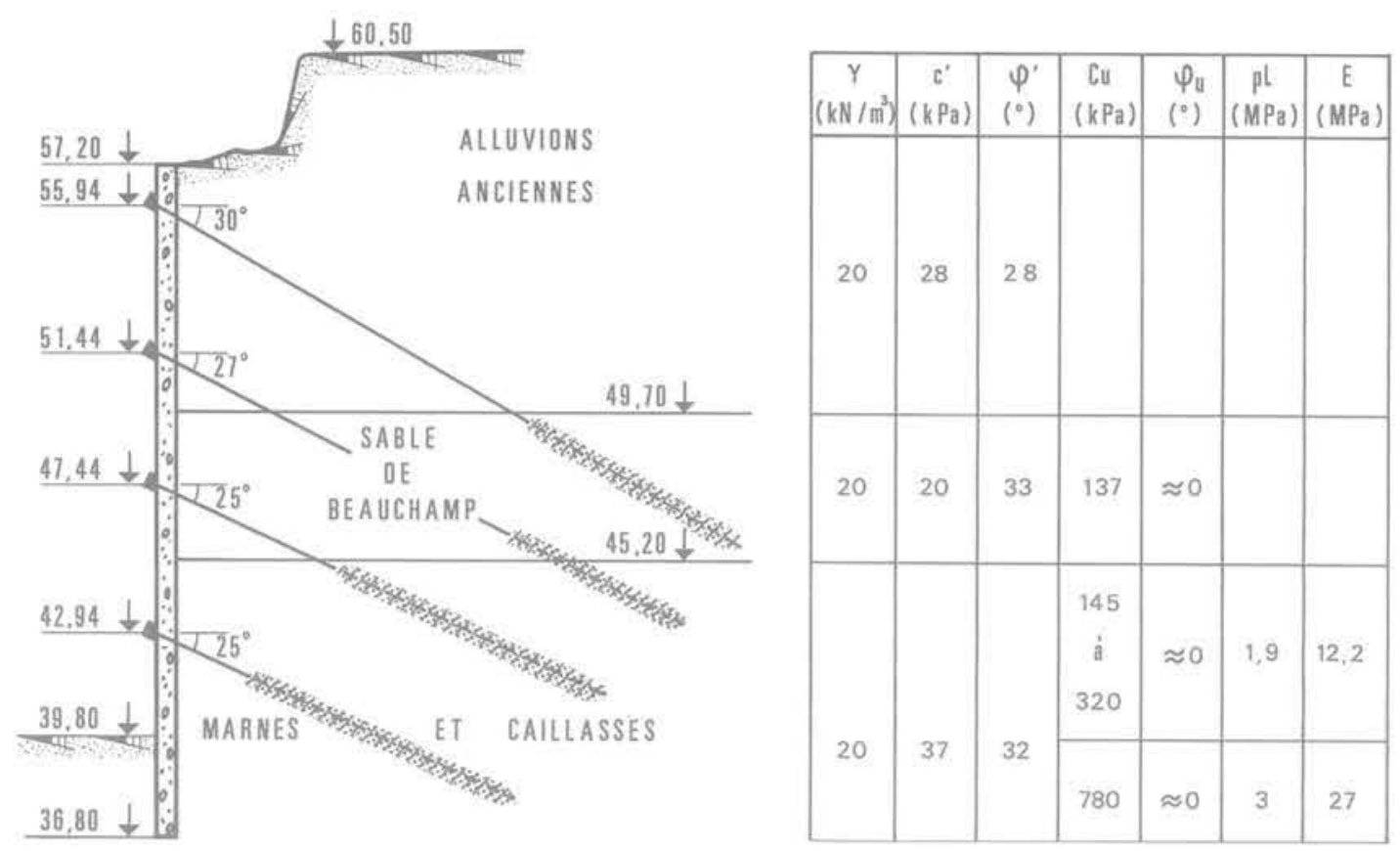

Fig. 1 Coupe de la paroi et caractéristiques du sol
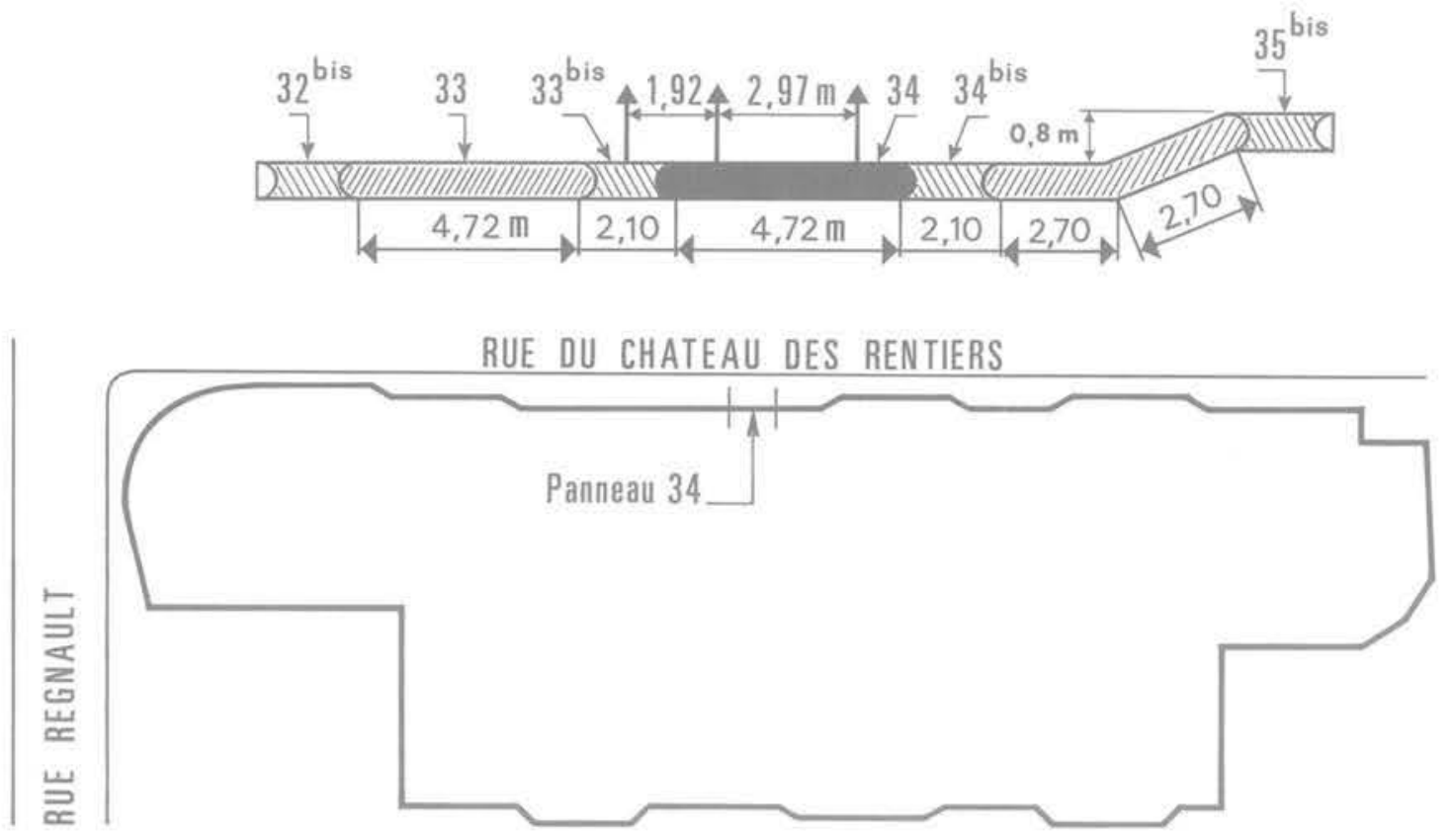

Fig. 2 Situation du panneau de mesure et détail de la paroi à proximité de celui-ci

Les caractéristiques mécaniques des échantillons extraits de ce sondage ont été déterminées en laboratoire à partir d'essais non consolidés - non drainés (UU), d'une part, et d'essais consolidés-drainés $(C D)$, réalisés sous contrepression, d'autre part. Les valeurs moyennes des paramètres de résistance au cisaillement ainsi obtenus sont indiquées figure 1 . On notera que, bien qu'aucune nappe ne baigne les formations étudiées, le degré de saturation du sable de Beauchamp et des marnes et caillasses est voisin de l'unité, ce qui explique que ces deux couches soient caractérisées par un angle de frottement apparent prati- quement nul.

Les essais UU effectués sur les échantillons prélevés dans les marnes et caillasses font apparaître une amélioration de la résistance au cisaillement non drainé dans la partie inférieure de la couche, c'est-à-dire au-dessous de la cote du fond de fouille de l'immeuble en fin de travaux. Cette amélioration est également mise en évidence par les essais pressio métriques effectués dans les marnes et caillasses devant le panneau de mesures, alors que le niveau d'excavation atteignait la base du sable de Beauchamp (fig. 1). 


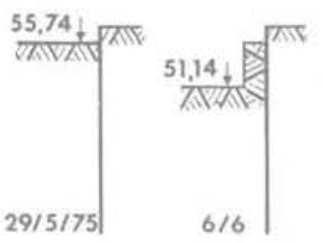

(1)

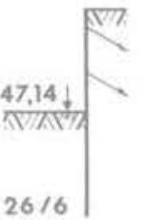

(6)

(2)

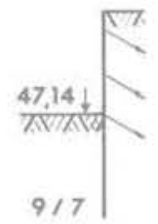

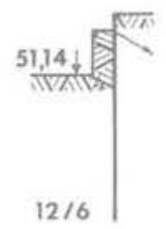
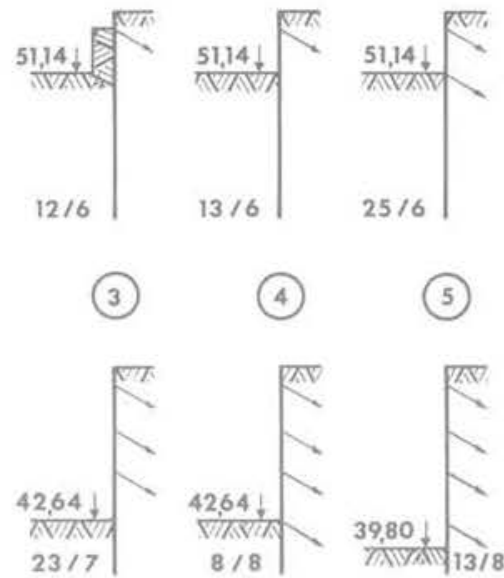

(8)

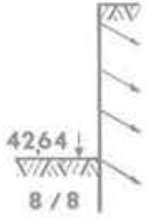

(9)
(5)

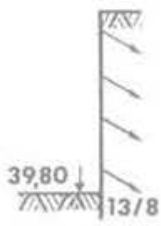

(10)

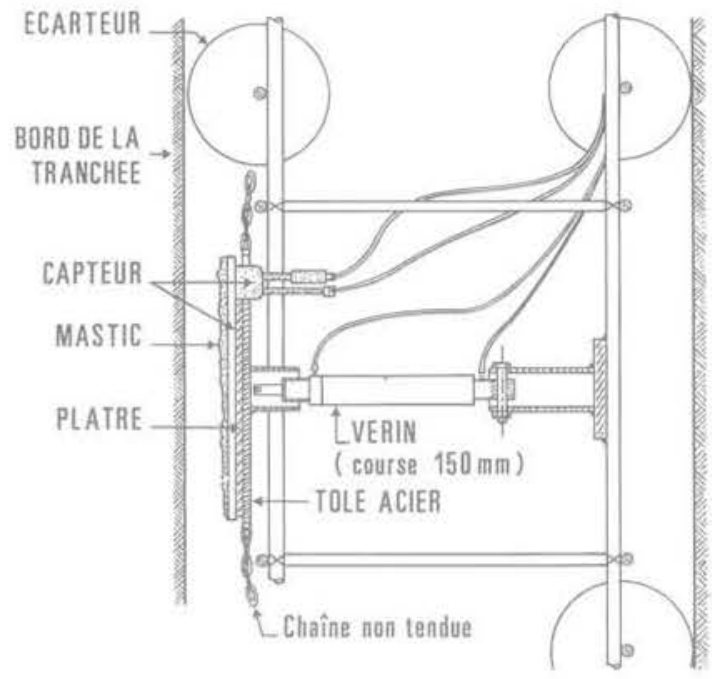

Fig. 3 Phases de travaux (phases de terrassement et de mise en tension des tirants)

\section{Déroulement des travaux}

Les phases de travaux peuvent se résumer en une succession d'excavations et de mises en tension des tirants jusqu'au niveau de la $4^{\circ}$ nappe, suivies d'une dernière excavation jusqu'au fond de fouille (fig. 3 ).

Les impératifs du chantier ne correspondant pas forcément à ceux qu'exigerait un bon déroulement des mesures, les terrassements ont été conduits de telle façon que le fond de fouille présentait des discontinuités importantes au voisinage immédiat du panneau expérimental, lors de certaines phases de travaux.

En particulier :

- lors de la réalisation de la plate-forme située au niveau de la $2^{\circ}$ nappe de tirants, une risberme a été laissée provisoirement devant le panneau expérimental :

- lors des terrassements de la plate-forme située au niveau de la $4^{\circ}$ nappe, le panneau expérimental n'a été dégagé que sur la moitié de sa longueur entre les niveaux des $3^{\circ}$ et $4^{\circ}$ nappes, ceci pendant une période d'une dizaine de jours.

Compte tenu de l'importance des déplacements mesurés en fin d'excavation au niveau de la tête $(45 \mathrm{~mm}$ pour certains panneaux), celle-ci a été confortée, par mesure de précaution, au moyen de butons inclinés prenant appui sur le fond de fouille. Le butonnage n'a intéressé que des panneaux suffisamment éloignés du panneau de mesures jusqu'à l'achèvement des terrassements devant ce dernier, mais un buton a été posé sur ce panneau deux jours après qu'il eut été dégagé sur toute sa hauteur libre.

\section{Equipement du panneau de mesures}

La pose d'appareils de mesure sur la paroi avait pour but : - de mesurer les efforts externes appliqués à la paroi, c'est-à-dire la pression des terres et les réactions d'ancrage :

- de déterminer les contraintes dans la paroi par l'intermédiaire des déformations relatives du béton:

- de déterminer les déplacements de la paroi à partir de mesures de déformée et de déplacement en tête.

La pression des terres a été mesurée au moyen de 33 capteurs de pression totale du type Glötzl dont les dimensions de la semelle étaient $200 \mathrm{~mm} \times 300 \mathrm{~mm}$. Chaque capteur était monté sur une plaque métallique adaptée à l'extrémité d'un petit vérin fixé sur les armatures de la cage (fig. 4). Une fois celle-ci descendue dans la tranchée, et avant bétonnage, les capteurs étaient mis au contact du sol au moyen des vérins commandés depuis la surface (la pression de contact était de $20 \mathrm{kPa}$ ). 28 capteurs ont été ainsi mis en place sur la face côté terrain, et 5 sur la face côté fouille, dans la partie en fiche (fig. 5).
Fig. 4 Dispositif utilisé pour l'application des capteurs de pression totale contre les parois de la tranchée

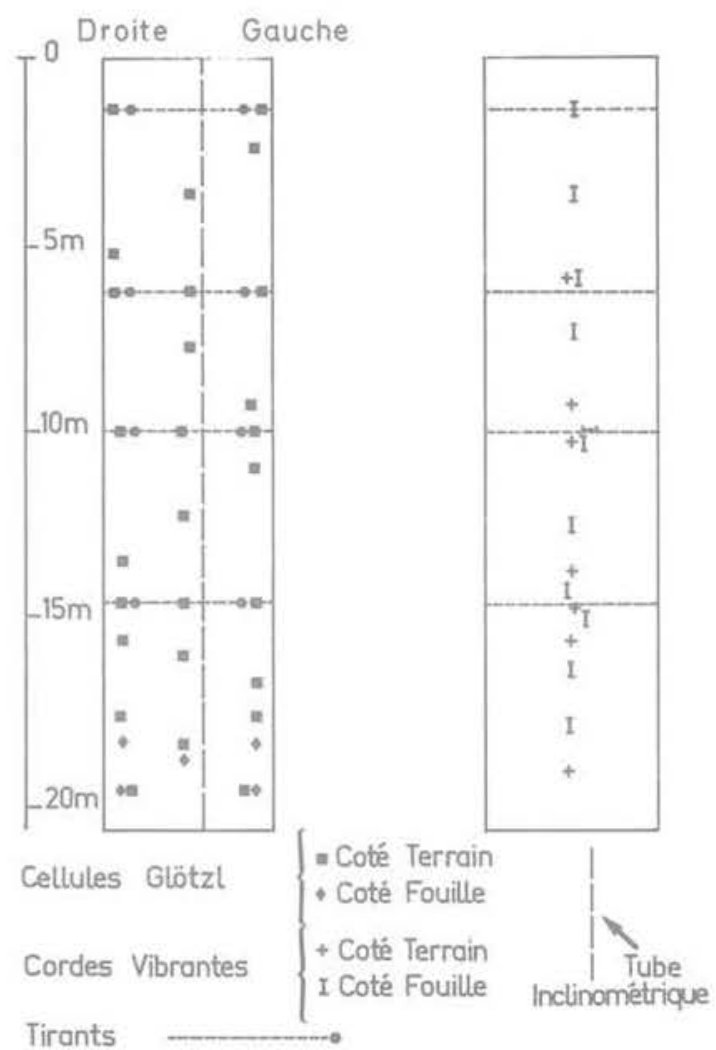

Fig. 5 Implantation des appareils équipant le panneau de mesure 
La pression du béton au cours du bétonnage a été mesurée au moyen d'un capteur Glötzl placé verticalement à $1,30 \mathrm{~m}$, au-dessus du fond de la tranchée.

Les déformations relatives du béton ont été mesurées au moyen de 20 extensomètres à corde vibrante du type Télemac, noyés dans le béton et mis en place de part et d'autre de laxe de la paroi dans les plans des armatures principales (fig. 5). Ces extensomètres étaient maintenus dans la position choisie au moyen de fils métalliques de petit diamètre attachés aux armatures (fig, 6).

Les tensions d'ancrage ont été mesurées au moyen de cales annulaires du type Glötzl, interposées entre la plaque d'ancrage et la tête du tirant. Une cale a été adaptée à chacun des 8 tirants du panneau de mesure, lors de sa mise en tension.

La déformée de la paroi a été déterminée au moyen d'un inclinomètre du type Soil Instruments, permettant de mesurer à tous niveaux inclinaison sur la verticale d'un tube inclinométrique noyé dans le béton de la paroi. La rotation de la tête de paroi a également été mesurée au moyen d'un clinomètre fixe.

Les données concernant le déplacement horizontal de la tête de paroi ont été obtenues:

- au moyen d'un tassomètre (distancemètre) à fil invar dont une extrémité était scellée au fond d'un forage subhorizontal de $15 \mathrm{~m}$. de profondeur réalisé au niveau de la tête de paroi ;

- au moyen de visées optiques effectuées à partir de points situés aux extrémités de la fouille, en dehors de son emprise (ces visées n'ont pas été effectuées sur le panneau de mesure mais sur des panneaux voisins).

Les appareillages de mesure ont presque tous fonctionné. Seuls un extensomètre implanté côté fouille au niveau de la $2^{\circ}$ nappe de tirants et la cale dynamométrique adaptée à l'un des tirants de la nappe supérieure n'ont fourni aucun résultat. En outre, les mesures inclinométriques ont dû être interrompues à la fin de la $2^{e}$ phase de travaux et n'ont pu être reprises que le 18 juillet, entre les $7^{\circ}$ et $8^{\circ}$ phases de travaux, avec un autre appareillage du même type. Les mesures effectuées avec le second appareil n'ont permis de déterminer que les déformées relatives de la paroi au cours des dernières phases de travaux.

\section{Résultats des mesures}

\subsection{Pression des terres}

Pression initiale sur la paroi.

Les premières mesures de pression des terres n'ont pu être effectuées qu'une semaine après le bétonnage, alors qu'une excavation d'environ $1,40 \mathrm{~m}$. de profondeur avait été creusée devant la paroi. Compte tenu de la faible profondeur de cette excavation, la distribution de la pression des terres le long de la paroi à ce stade des travaux peut être considérée comme la distribution initiale.

La pression $\sigma_{h}$ croit de facon sensiblement linéaire avec la profondeur z comptée à partir de la tête de paroi (fig. 7). alors que le rapport $K=\frac{\sigma_{h}}{\gamma z}$ décroît avec la profondeur
(fig. 8):

- Alluvions anciennes : $0,39<\mathrm{K}<0,75, \mathrm{~K}_{\text {moy }}=0,6$

- Sable de Beauchamp : $0,37<K<0,69, K_{\text {moy }}=0,49$

- Marnes et caillasses : $0,25<\mathrm{K}<0,70, \mathrm{~K}_{\text {moy }}=0,41$

En revanche les valeurs du rapport de la contrainte horizontale mesurée à la contrainte verticale $\gamma_{z 0}$ qui s'exerçait au niveau de mesure avant tout terrassement se placent, pour la plupart, dans l'intervalle 0,20-0,50 (moyenne générale : 0,33 ) quelle que soit la profondeur considérée (fig. 8).

II pourrait donc sembler, en première analyse, que l'ouverture de la fouille creusée à partir du terrain naturel pour dégager la plate-forme de travail, ainsi que la réalisation de la paroi, n'aient pas modifié sensiblement l'état initial

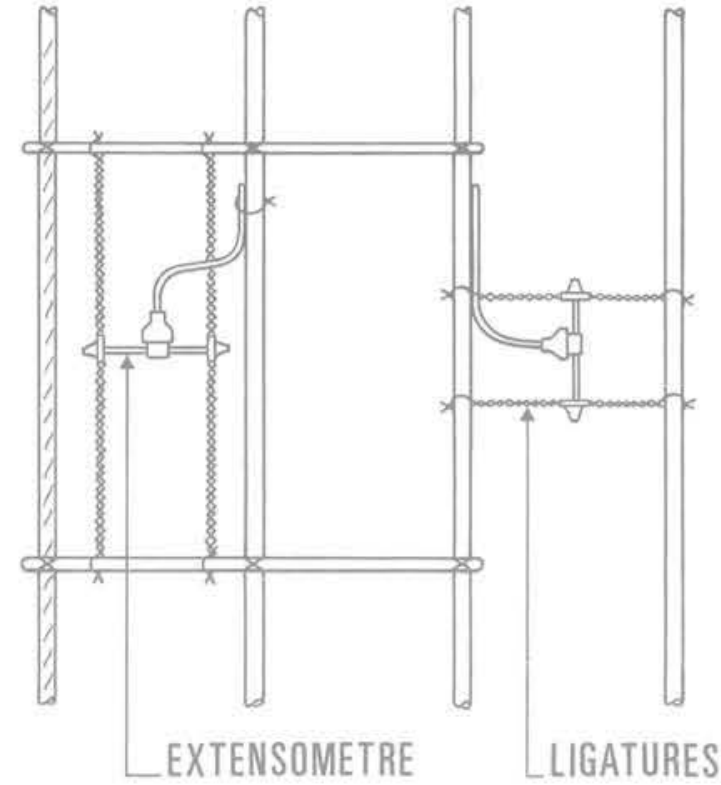

Fig. 6 Dispositif de fixation des extensomètres sur la cage d'armature

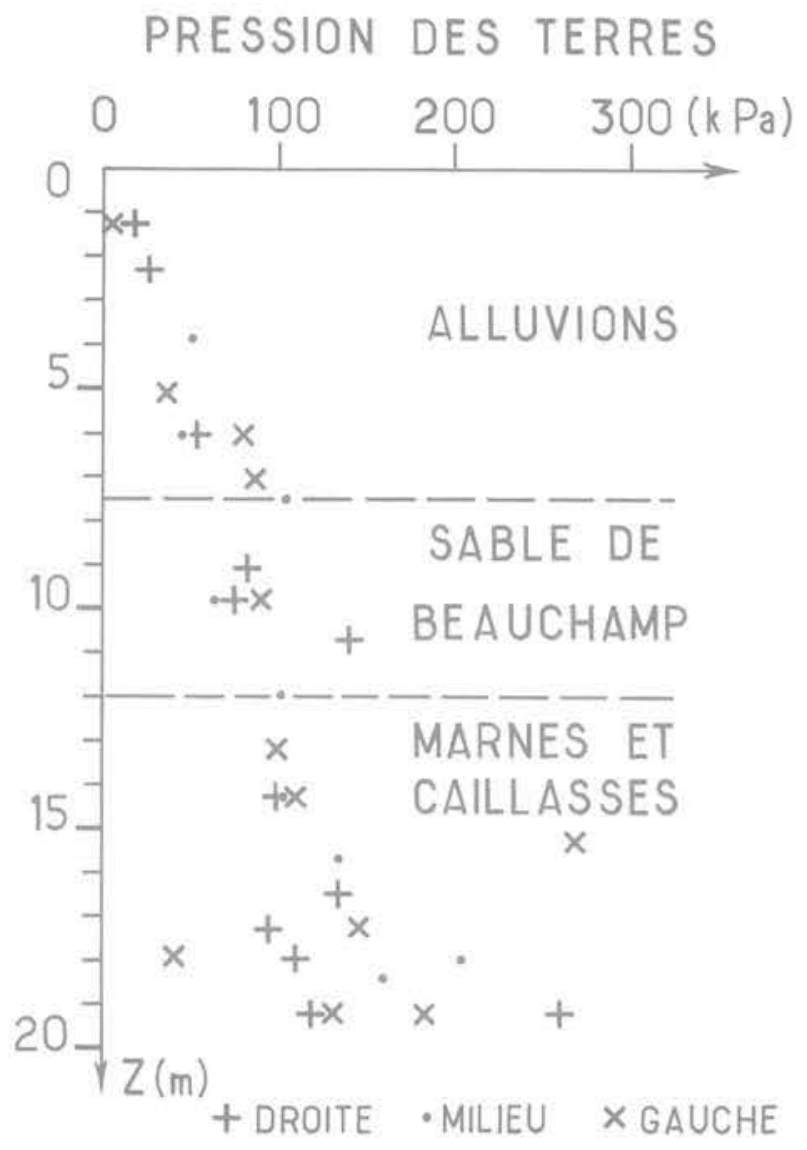

Fig. 7 Distribution initiale de la pression des terres 


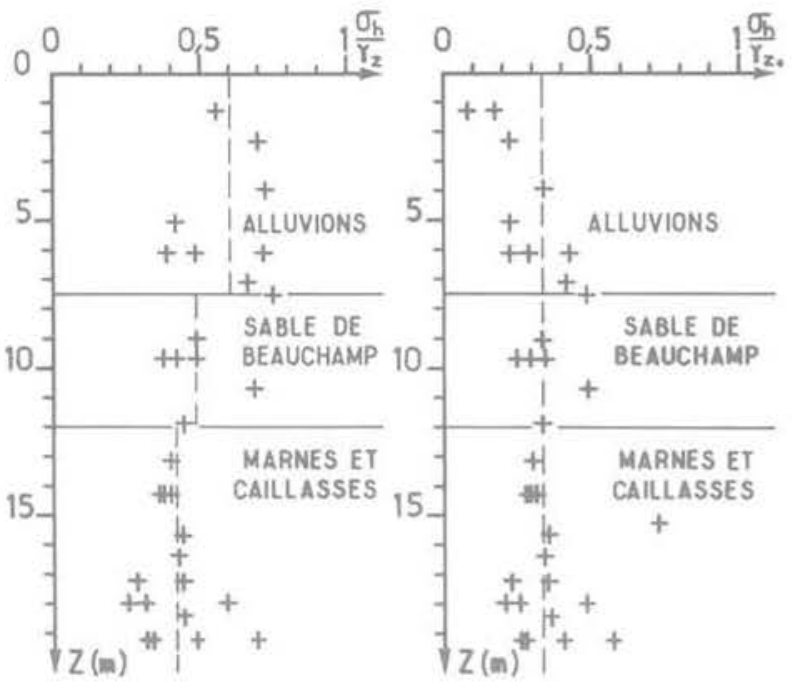

Fig. 8 Variations en fonction de la profondeur des rapports $\frac{\sigma_{h}}{\gamma_{z}}$ et $\frac{\sigma_{h}}{\gamma_{z o}}, z$ représentant la profondeur par rapport à la tête de paroi et $z_{0}$ la profondeur par rap. port à la surface initiale du sol $\left(z_{0}-z=3,30 \mathrm{~m}\right)$

des contraintes horizontales dans le massif. Dans ces conditions le coefficient de pression des terres au repos serait en moyenne de: 0.33 .

En fait, l'examen des sollicitations imposées au sol montre que l'analyse précédente ne peut être retenue.

En effet,

- le sol initialement au repos est décomprimé latéralement lors de l'ouverture de la tranchée:

- le béton liquide déversé dans la tranchẻe impose tout d'abord une distribution hydrostatique de la pression horizontale, mais celle-ci est très vite perturbée par les phénomènes liés à la prise du béton ainsi que le montre l'évolution de la pression horizontale du béton en pied de paroi mesurée au moyen d'un capteur Glötzl (fig. 9).

Dans ces conditions, il semble assez peu vraisemblable que la pression initiale mesurée le long de la paroi soit en relation simple avec le coefficient de pression du sol au repos.

Pression sur la paroi à la fin des terrassements.

La distribution de la pression des terres mesurée sur le pourtour de la paroi après la dernière phase de terrassement, quelques heures avant la pose du buton placé au droit du panneau de mesures (14 août), est représentée figure 10, ainsi que les diagrammes limites de pression des terres, déterminés par la méthode de Caquot-Kérisel sur la base des paramètres de cisaillement drainé des différentes couches, en tenant compte d'une inclinaison des contraintes sur l'horizontale, égale à $\hat{\delta}_{\mathrm{a}}=\frac{2}{3} \sigma^{\prime}$ dans le cas de la poussée et à $\dot{\delta}_{p}=-\frac{2}{3} \emptyset$ dans le cas de la butée.

On remarque que, sur toute la hauteur de la paroi, la pression sur la face arrière est largement supérieure à la poussée limite ainsi calculée (poussée à long terme). On notera que cette dernière représente la valeur maximale de la poussée mobilisable, la poussée à court terme étant considérablement plus faible, mais compte tenu du mode d'ancrage de la paroi il n'y avait a priori aucune raison pour que le sol soit en état d'équilibre limite.

Le petit nombre de capteurs implantés sur la face avant ne permet pas une détermination précise du diagramme de pression du sol sur la hauteur en fiche. Les pressions mesurées au niveau de deux capteurs situés à $0,50 \mathrm{~m}$. et $1.10 \mathrm{~m}$. au-dessous du fond de fouille correspondent respectivement à des taux de mobilisation de la butée limite théorique de 0,72 et de 0,82 , les trois autres capteurs

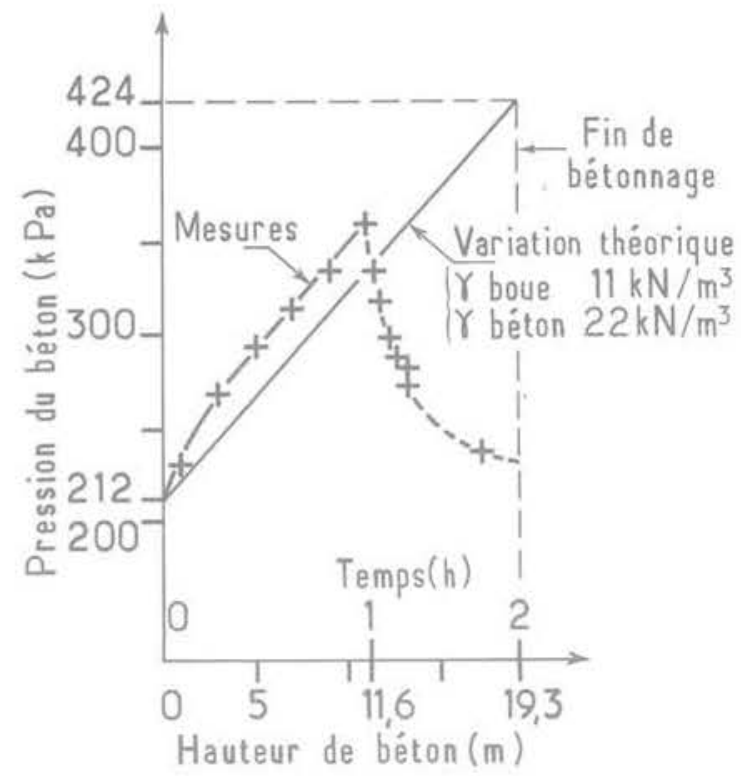

Fig. 9 Evolution de la pression du béton liquide à 1,30 $\mathrm{m}$ au-dessus du fond de tranchée au cours du bétonnage du panneau

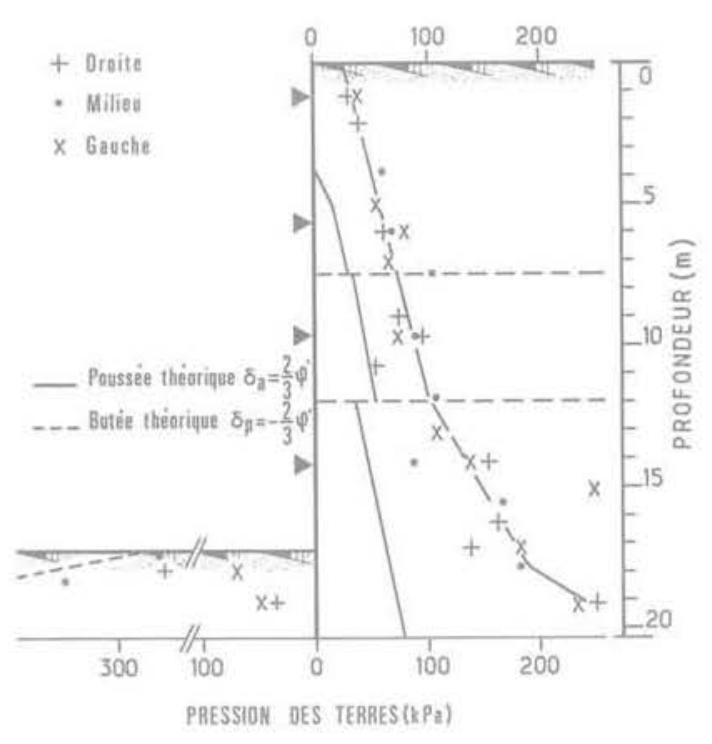

Fig. 10 Distribution de la pression des terres à la fin des terrassements

indiquant des pressions beaucoup plus faibles. Compte tenu d'un remaniement probable du fond de fouille (action des engins de terrassement et décompression du sol) ayant pour effet de réduire la résistance du sol, il semblerait que celui-ci soit dans un état voisin de l'équilibre limite de butée sur approximativement le tiers supérieur de la fiche et en équilibre élastique sur les deux tiers inférieurs.

Les pressions élevées mesurées à la base de la paroi sur la face arrière et les faibles pressions mesurées au même niveau sur la face avant, suggèrent l'existence d'une contre-butée en pied de paroi. 
Variations de la pression sur la paroi au cours des phases intermédiaires de travaux.

Les variations moyennes de la pression du sol (moyenne des variations mesurées le long des trois files de cellules) entre la première phase assimilée à l'état initial et la fin des travaux sont représentées figure 11. L'allure et l'amplitude de ces variations dans la partie en fiche sont bien cohérentes avec l'hypothèse d'une contrebutée en pied de paroi. Les variations constatées sur la hauteur hors fiche sont, en revanche, très faibles puisqu'elles ne dépassent pas $30 \mathrm{kPa}$. Ces variations globales sont la résultante de variations de signe différent, qui se sont produites lors des principales phases de travaux (terrassements, mises en tension des tirants) ainsi qu'entre ces phases.

Les phases de terrassement (fig. 12) se traduisent par une diminution de la pression du sol sur la face arrière au-dessus du niveau de terrassement et dans certains cas par une mise en contrebutée du sol, au-dessous de ce niveau. On note généralement une légère augmentation de la tension des tirants les plus proches du niveau d'excavation.

Une mise en tension (fig. 13) se traduit par une augmentation de la pression du sol au contact de la face arrière, de part et d'autre du niveau de la nappe mise en service, l'augmentation de pression étant maximum au niveau de cette nappe. On note également une légère diminution des tensions des tirants des nappes supérieures.

On a également constaté des variations sensibles de la pression du sol au cours des périodes de "repos " séparant les principales phases de travaux (fig. 14). Ces variations semblent être dues en grande partie aux travaux réalisés de part et d'autre du panneau de mesures au cours de ces périodes, un panneau ne travaillant pas indépendamment des panneaux voisins. Un phénomène de déformation différée du sol peut également intervenir dans ces variations.

\section{Dispersion des mesures.}

Les mesures de pression des terres peuvent être assez fortement dispersées, notamment celles effectuées à un même niveau de la paroi. Cette dispersion n'est pas liée seulement à un "vrillage " du panneau de mesures dâ aux discontinuités de terrassement (vrillage qui modifie la distribution de la pression du sol), puisqu'elle apparaît également lors des mesures initales (fig. 7). Elle tient également aux conditions de contact entre les capteurs et le sol qui peuvent varier d'un capteur à l'autre en raison des différences d'épaisseur du cake, du remaniement plus ou moins grand du sol, de la présence de gros éléments.

\subsection{Tension d'ancrage}

L'évolution des tensions des sept tirants sur lesquels des mesures ont pu être effectuées est représentée figure 15 en fonction du temps. La comparaison de la tension de service théorique (tension de précontrainte théorique) aux différents niveaux d'ancrage avec les tensions effectivement mesurées à ces niveaux ne fait pas apparaître de divergences sensibles entre ces valeurs en ce qui concerne la première nappe d'ancrage. En revanche, les tensions mesurées dans les tirants de la $2^{a}$ nappe se situent toujours au-dessus de la valeur théorique de service lécart maximal $24 \%$ ), alors que celles obtenues pour les tirants des nappes inférieures se situent en permanence au-dessous (écart maximal $-27 \%$ pour le tirant droit de la $4^{\circ}$ nappe). On notera que les divergences constatées entre les tensions de service théoriques et les tensions mesurées apparaissent dès les premières mesures suivant la mise en tension et évoluent peu par la suite.

On remarque également des différences très sensibles entre les tensions des tirants de la $2^{\circ}$ nappe et surtout entre celles des tirants de la $4^{\circ}$ nappe. Ces différences semblent dues en grande partie au « vrillage » du panneau de mesures lié aux conditions d'exécution des travaux.

\subsection{Déformation relatives du béton}

Dans l'hypothèse oú le béton de la paroi travaille en sec-
VARIATIONS DE PRESSION ( $\left.K P_{2}\right)$ SUR LA FACE ARRIERE

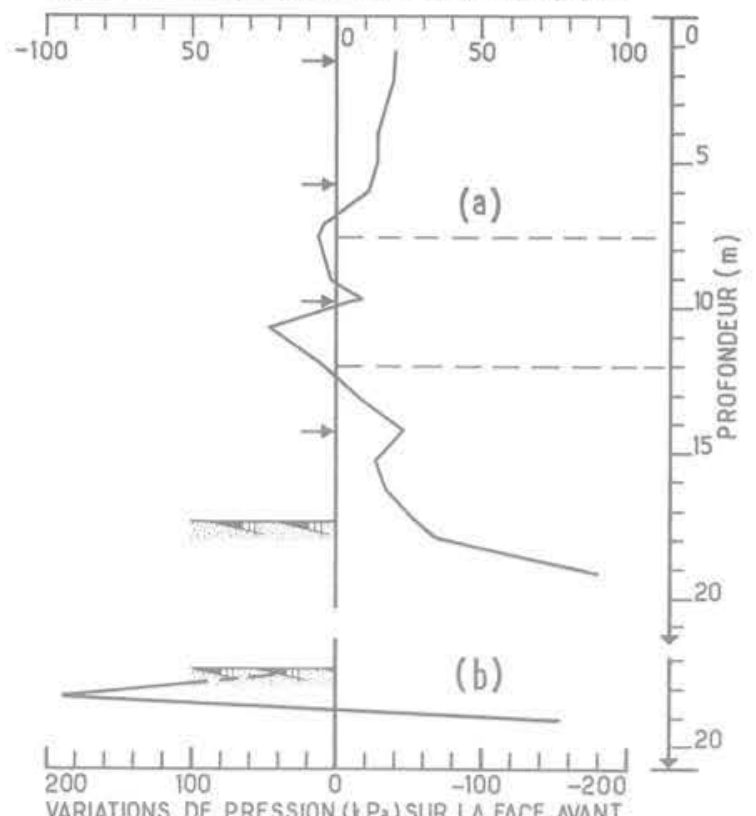

Fig. 11 Variations moyennes de la pression des terres au cours des travaux :

a) sur la face arrière

b) sur la face avant

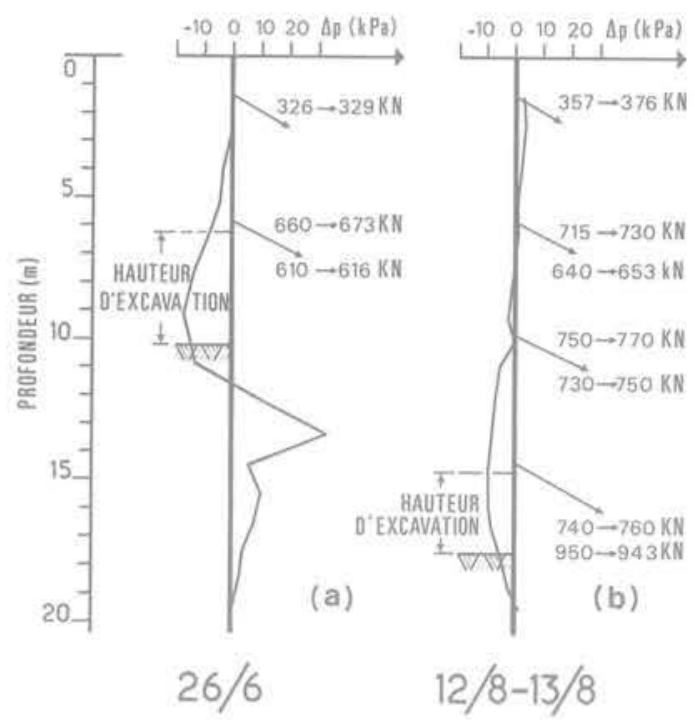

Fig. 12 Incidence des terrassements sur la pression du sol sur la face arrière de la paroi et sur les tensions $d^{\prime}$ ancrage $\left({ }^{*}\right)$ :

a) terrassement entre les niveaux des 2ème et 3ème nappes

b) terrassement entre le niveau de la 4ème nappe et le fond de fouille

(*) Les valeurs indiquées au niveau de chaque nappe sont celles des tensions d'ancrages mesurées au niveau des deux tirants correspondants du panneau d'essai au début (valeurs de gauche) et à la fin (valeurs de droite) de l'opération ou de la période considérée. Lors de la mise en tension d'une nappe, seules sont reportées au niveau de cette dernière les tensions mesurées à la fin de l'opération 

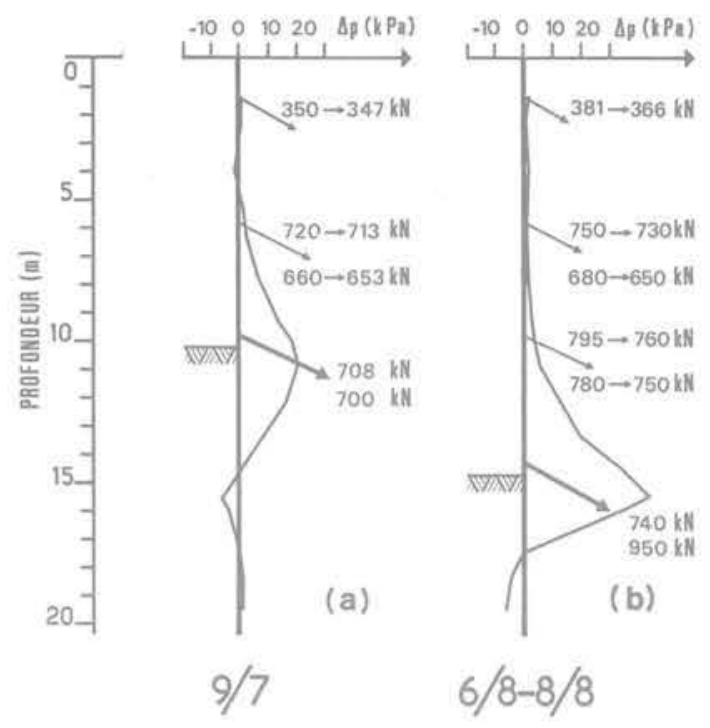

$6 / 8-8 / 8$

Fig. 13 Incidence de la mise en tension d'une nappe sur la pression du sol sur la face arrière de la paroi et sur les tensions des autres tirants ("):

a) mise en tension de la 3ème nappe

b) mise en tension de la 4ème nappe

tion homogène les déformations relatives $\varepsilon_{\mathrm{T}}$ et $\varepsilon_{F}$ mesurées respectivement côté terrain et côté fouille dans le plan des armatures principales à un niveau donné peuvent être exprimées en fonction du moment fléchissant $M$ et de l'effort normal $\mathrm{N}$ par mètre de paroi, s'exerçant à ce niveau. On a alors,

$$
\begin{aligned}
& \varepsilon_{T}=-\frac{N}{E e}-\frac{M d}{E l} \\
& \varepsilon_{F}=-\frac{N}{E e}+\frac{M d}{E l}
\end{aligned}
$$

avec les notations et conventions de signes suivantes:

$$
\begin{aligned}
& \text { e, épaisseur de la paroi } \\
& \text { E. module d'Young de béton }
\end{aligned}
$$

$1=\frac{\mathrm{e}^{3}}{12}$, inertie de la paroi par métre linéaire

d. distance de l'axe de la paroi aux plans des armatures principales $(d=0,20 \mathrm{~m}$.).

$M$ est compté positivement lorsque la concavité de la paroi est dirigée vers l'arrière (côté terrain),

Nest compté positivement lorsque la paroi est comprimée axialement. traction.

$\varepsilon_{\mathrm{T}}$ et $\varepsilon_{\mathrm{F}}$ sont positifs lorsque le béton travaille en

La transformation de ces relations conduit:

$$
\begin{aligned}
& \varepsilon_{\mathrm{M}}=\frac{1}{2}\left\langle\varepsilon_{\mathrm{F}}-\varepsilon_{\mathrm{T}}\right)=\frac{\mathrm{Md}}{\mathrm{El}}=\frac{12 \mathrm{Md}}{\mathrm{Ee}^{3}}=\lambda \mathrm{M} \\
& -\varepsilon_{\mathrm{N}}=-\frac{1}{2}\left(\varepsilon_{\mathrm{F}}+\varepsilon_{\mathrm{T}}\right)=\frac{\mathrm{N}}{\mathrm{Ee}}=\mu \mathrm{N}
\end{aligned}
$$

II s'ensuit que si l'on reporte en fonction de z sur un même diagramme les variations de $\varepsilon_{\mathrm{F}}$ et de $-\varepsilon_{\mathrm{T}}$. l'abscisse de la courbe moyenne est proportionnelle à la valeur du moment fléchissant. D'autre part, la différence $-2 \varepsilon_{N}$ des abscisses des courbes $-\varepsilon_{\mathrm{T}}$ et $\varepsilon_{\mathrm{F}}$ est égale à un facteur près à la valeur de l'effort normal.

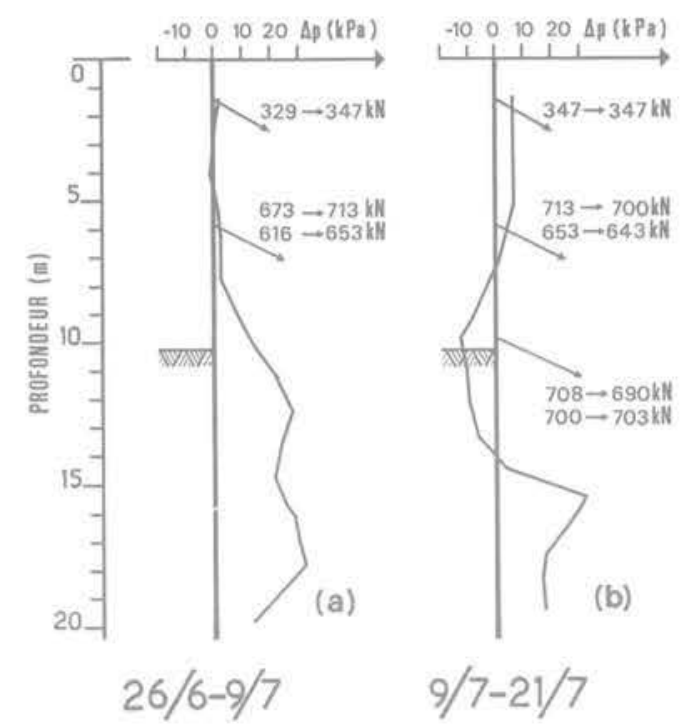

Fig. 14 Variations des tensions d'ancrage (*) et de la pression sur la face arrière de la paroi au cours de périodes de "repos》:

a) entre les 6ème et 7ème phases de travaux

b) entre les 7ème et 8ème phases de travaux

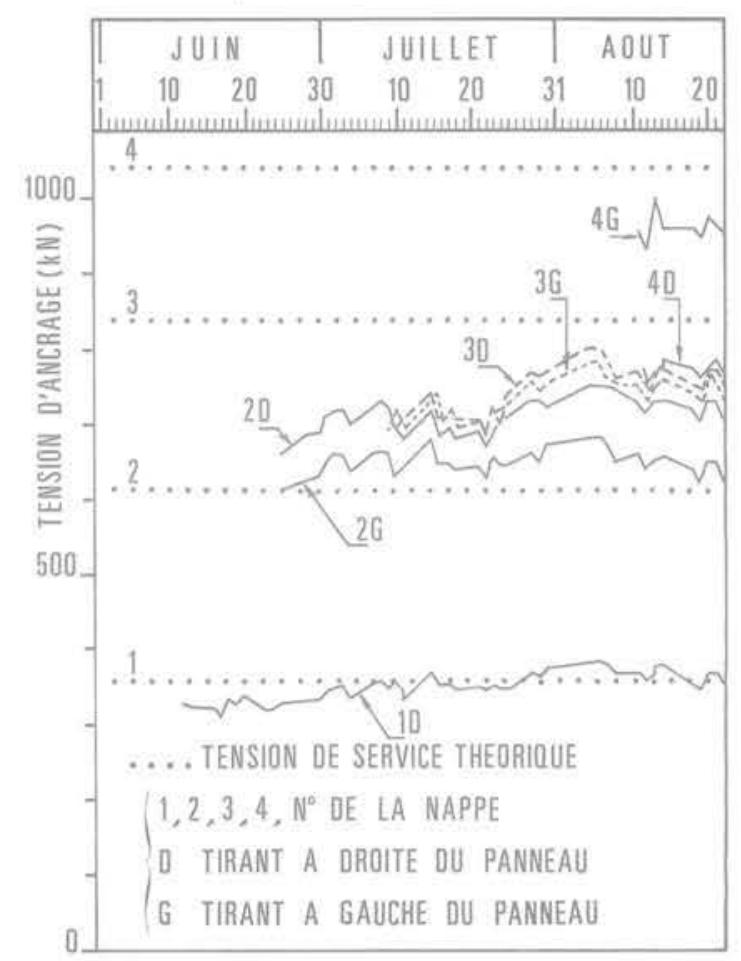

Fig. 15 Variation des tensions d'ancrage pendant la durée des travaux 

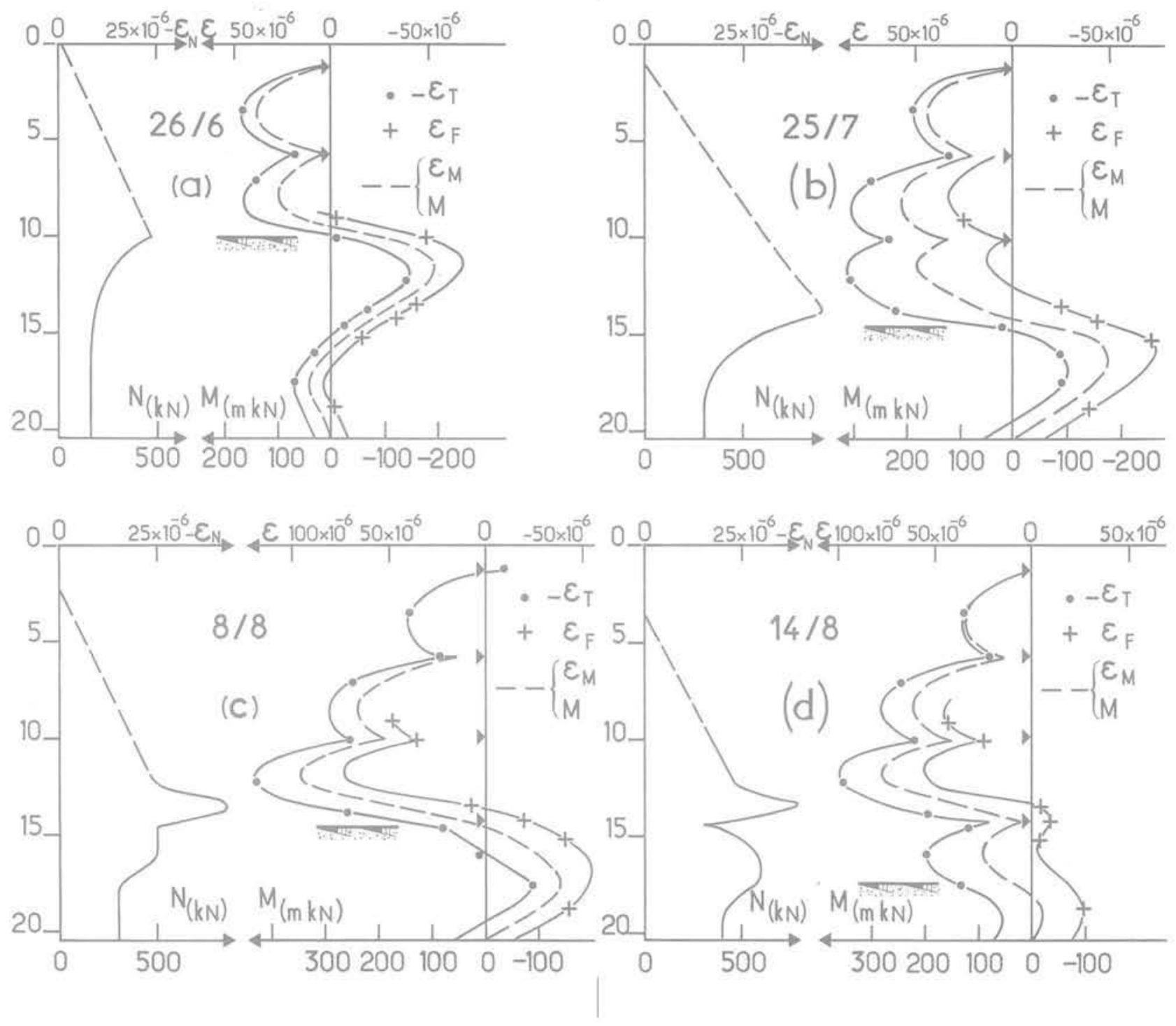

Fig. 16 Diagrammes des déformations relatives du béton :
a) après terrassement jusqu'à la 3ème nappe (Gème phase)
b) après terrassement jusqu'à la 4ème nappe (8ème phase)
c) après mise en tension de la 4ème nappe (9ème phase)
d) après terrassement jusqu'au fond de fouille (10ème phase)

Les variations de $\varepsilon_{T}, \varepsilon_{F}, \varepsilon_{M}, \varepsilon_{N}$ sur la hauteur de la paroi sont représentées figure 16 pour 4 phases de travaux. On notera :

- qu'en l'absence de mesures côté fouille sur les $9 \mathrm{~m}$ supérieurs de la paroi, les courbes $\varepsilon_{M}$ dans cette partie du panneau ont dû être déterminées sur la base des valeurs de la rotation $\omega$ de la tête de paroi indiquées par le clinomètre et des valeurs $\omega_{0}$ de la rotation du pied de paroi résultant des mesures inclinométriques effectuées à partir du 18 juillet $\left(\omega_{0}\right.$ a été supposé nul lors des premières phases de travaux). Elles ont été tracées par approximations successives de façon à ce que la fonction $\varepsilon_{M}(z)$ satisfasse à la relation :

$$
\omega=\omega_{0}+\int_{0}^{h} \frac{\varepsilon_{M}(z)}{d} d z
$$

Les valeurs de $\varepsilon_{N}$ à la partie supérieure de la paroi ne peuvent être obtenues qu'à partir de celles de $\varepsilon_{M}$ (au moyen de la relation $\varepsilon_{N}=\varepsilon_{\tau}+\varepsilon_{M}$ ) et, de ce fait, ne peuvent être considérées que comme des ordres de grandeur. C'est pourquoi, sur les 9 mètres supérieurs, on a représenté la courbe $\varepsilon_{N}(z)$ par une droite qui ne suit quapproximativement les variations de $\varepsilon_{\mathrm{T}}+\varepsilon_{\mathrm{M}}$.

- que les mesures effectuées pendant les 15 jours suivant le bétonnage de la paroi ayant mis en évidence des déformations relativement importantes qui ne pouvaient manifestement pas être dues aux efforts appliqués à la paroi mais résultaient du retrait du béton, les valeurs de $\varepsilon$ prises en compte pour l'étude de la paroi sont des valeurs corrigées n'intégrant pas les déformations parasites constatées. 


\subsection{Déplacements de la paroi}

Le déplacement horizontal de la tête de paroi par rapport au scellement du tassomètre croît assez régulièrement au cours de la fouille pour atteindre $15 \mathrm{~mm}$. en fin d'excavation (fig. 17). Les mesures par visées optiques du déplacement de repères placés en tête de panneaux voisins du panneau de mesures indiquent des déplacements beaucoup plus importants (fig. 17) mettant ainsi en évidence un mouvement du scellement du tassomètre. Bien que les mesures par visées optiques n'aient été réalisées qu'au cours des dernières phases de travaux (au mieux à partir de la $5^{\circ}$ phase), il est possible d'en déduire une limite inférieure du déplacement absolu de la tête de paroi en fin de travaux, en admettant que, pendant la période couvrant les 5 premières phases, les déplacements sont de l'ordre de ceux indiqués par le tassomètre. Dans cette hypothèse le déplacement de la tête de paroi en fin de travaux est d'au moins $45 \mathrm{~mm}$., c'est-à-dire supérieur à $2 / 1000$ de la hauteur de la paroi. Il s'ensuit que le scellement du tassomètre situé à $15 \mathrm{~m}$. en arrière de la paroi (c'est-à-dire à l'extérieur du coin de poussée théorique) se déplace vers la fouille d'au moins $30 \mathrm{~mm}$. Cela implique que les déformations du sol à l'arrière de la paroi intéressent une zone étendue dont la largeur est vraisemblablement très supérieure à la profondeur d'excavation. L'importance de la zone concernée par la décompression du sol à l'arrière d'un soutènement de grande hauteur a d'ailleurs été mise en évidence par des études théoriques basées sur le calcul aux éléments finis (Deroy, 1975).

Les diagrammes de la figure 17 fvisées optiques et tassomètre) mettent en évidence des mouvements de la tête de paroi entre deux phases de travaux intéressant directement le panneau sur lequel sont effectuées les mesures. Ces mouvements sont à rapprocher des variations de pression des terres constatées entre deux phases de travaux, et ont apparemment la même origine.

Les mesures inclinométriques effectuées à partir du 18 juillet n'ont permis de déterminer les déformations de la paroi qu'au cours des dernières phases de travaux. Les déformations de la paroi depuis le 18 juillet sont représentées figure 18 , à 3 stades des travaux : après terrassement jusqu' au niveau de la $4^{\mathrm{e}}$ nappe $(28 / 7)$, après mise en tension de celle-ci $(11 / 8)$ et après terrassement jusqu'au fond de fouille $(13 / 8)$. Les mesures de déplacement (tassomètre et mesures optiques) n'indiquant aucun recul de la tête de paroi vers l'arrière, l'examen des déformées obtenues le 11 aout et le 13 août montre qu'entre ces deux dates, c'est-à-dire lors de la dernière phase de terrassement, la partie inférieure de la paroi s'est déplacée d'au moins $4 \mathrm{~mm}$ vers la fouille. Un tel déplacement est cohérent avec les variations de la pression des terres constatées à ce stade du chantier (diminution sensible de la pression sur la face arrière à la partie inférieure de la paroi).

\section{Discussion et interprétation des mesures}

Les paramètres mesurés n'étant pas indépendants les uns des autres. les relations existant entre eux permettent d'apprécier la validité des résultats obtenus et, le cas échant, de déterminer la valeur de grandeurs difficilement mesurables, telles que le module d'Young du béton, le frottement sol-paroi, etc.

\subsection{Relations générales entre les paramètres mesures}

Les relations entre les paramètres déterminant le comportement de l'ouvrage expriment l'équilibre d'un élément de paroi de longueur unité, limité à sa partie supérieure par la tête de paroi et à sa partie inférieure par une section de cote z située au-dessus du fond de fouille, ou éventuellement un peu au-dessous, pourvu que l'action du sol sur la face avant puisse être négligée (en raison du nombre très limité de capteurs de pression implantés sur la face avant, i) n'a pas été possible d'étudier l'équilibre global de la paroi). A cet élément sont appliqués (fig. 19):

- la pression des terres $p$.

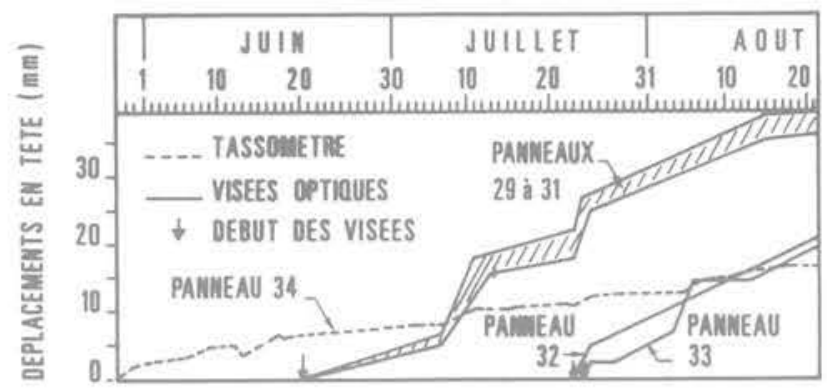

Fig. 17 Déplacements horizontaux de la tête de paroi au cours des terrassements

\section{DEFORMATIONS ( $\mathrm{mm}$ )}

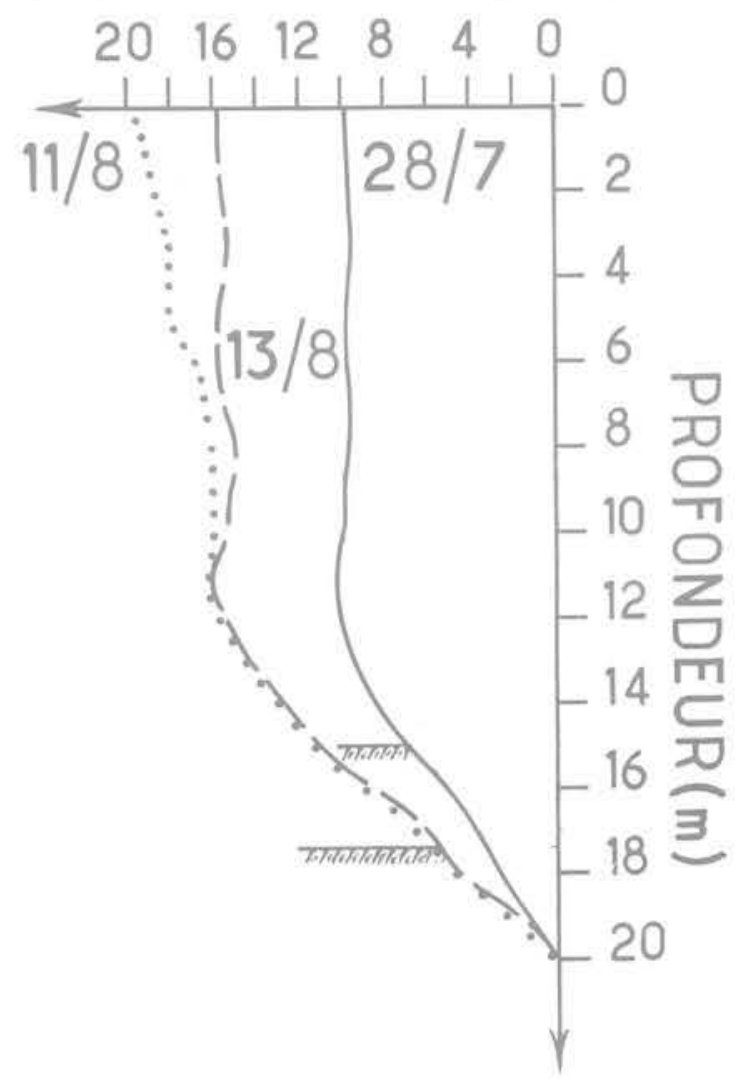

Fig. 18 Déformations de la paroi depuis le 18 juillet à trois stades ultérieurs des travaux

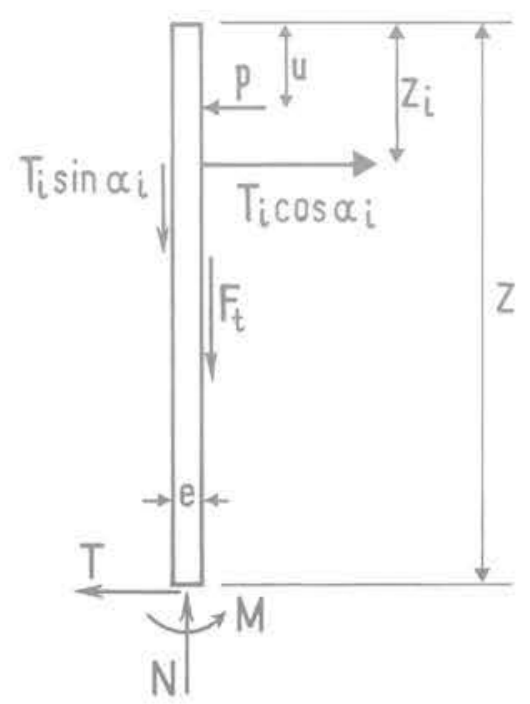

Fig. 19 Efforts appliqués à un élément de paroi 
- les composantes horizontales $T_{i} \cos \alpha_{i}$ et verticales $T_{i} \sin$ des tensions d'ancrages,

- la résultante $F_{t}$ des efforts de frottement du sol sur la face arrière,

- l'effort normal N, l'effort tranchant $T$ et le moment fléchissant $\mathrm{M}$ dans la section de cote $\mathrm{z}$,

L'équilibre de l'élément conduit aux relations:

$$
N=F_{i}+\sum_{1}^{n} T_{i} \sin \alpha_{i}
$$

n étant le nombre de nappes d'ancrage intéressant l'élément considéré.

$$
T=\sum_{1}^{n} T_{i} \cos \alpha_{i}-\int_{0}^{z} p d z
$$

$M=-\int_{0}^{z} p(z-u) d u+\sum_{1}^{n} T_{i} \cos \alpha_{i}\left(z-z_{i}\right)-\frac{e}{2} \sum_{1}^{n} T_{i} \sin \alpha_{i}+\frac{e F_{t}}{2}$

ou encore, en combinant (6) et (8),

$M=-\int_{0}^{z} p(z-u) d u+\sum_{1}^{n} T_{i} \cos \alpha_{i}\left(z-z_{i}\right)-e_{1}^{n} T_{i} \sin \alpha_{i}+\frac{e N}{2}$

$M$ et $N$ sont par ailleurs exprimés en fonction de $\varepsilon_{M}$ et $\varepsilon_{N}$. par l'intermédiaire des relations (3) et (4).

\subsection{Equilibre de la paroi}

Entre deux niveaux d'ancrage, la relation entre le moment fléchissant et l'effort tranchant s'écrit:

$$
\frac{d M}{d z}=T+\frac{e}{2} \frac{d F_{t}}{d z}=T+\frac{e}{2} \frac{d N}{d z}
$$

Aux points de moment fléchissant maximum

$$
\left(\frac{\mathrm{dM}}{\mathrm{dz}}=\frac{\mathrm{d} \varepsilon_{\mathrm{M}}}{\mathrm{dz}}=0\right)
$$

de cote $z_{m j} j$ désignant le numéro d'ordre des points rencontrés à partir de la tête de paroi, les relations (7) et (10) conduisent à :

$T=\sum_{1}^{j} T_{i} \cos \alpha_{i}-\int_{0}^{z_{m i}} p d z=-\frac{e}{2}\left(\frac{d N}{d z}\right)_{z}=z_{m i}=-\frac{e}{2} \cdot \frac{d N_{i}}{d z}$

soit,

$$
\int_{0}^{z_{m j}} p d z=\sum_{i}^{j} T_{i} \cos \alpha_{i}+\frac{e}{2} \frac{d N_{j}}{d z}
$$

Si le terme $\frac{e}{2} \frac{d N_{j}}{d z}$ est faible ou varie peu d'un point de moment fléchissant au suivant, la relation (11) conduit à :

$$
\int_{z_{m j-1}}^{z_{m i}} p d z=T_{i} \cos \alpha_{j}
$$

relation qui exprime que la pression des terres s'exerçant entre deux points consécutifs de moment fléchissant maximum est équilibrée par la composante horizontale des tensions des tirants de la nappe située entre ces deux points.

Au point de moment fléchissant nul $\left(\varepsilon_{M}=0\right)$ de cote $z_{0}$ qui se situe le plus souvent à proximité du fond de fouille à partir de la $3^{e}$ phase de travaux, la relation (9) s'écrit en négligeant ses deux derniers termes qui ont une influence secondaire :

$$
\int_{0}^{z_{0}} p\left(z_{0}-u\right) d u=\sum_{1}^{n} T_{i} \cos \alpha_{i}\left(z-z_{i}\right)
$$

L'étude de l'équilibre de la paroi a consisté à vérifier pour les principales phases de travaux:

- l'équilibre des forces appliquées au-dessus de chacun des points de moment fléchissant maximum situés dans la partie hors fiche, au moyen de la relation (12);

- l'équilibre des forces appliquées entre deux points consécutifs de moment fléchissant maximum situés dans la partie hors fiche, au moyen de la relation (13)

- l'équilibre des moments par rapport au point de moment fléchissant nul, des forces appliquées au-dessus de celui-ci, au moyen de la relation (14).

La tension moyenne d'ancrage $T_{\text {i }}$ par mètre de panneau au niveau de la nappe i a été évaluée en admettant qu'un tirant équilibrait les efforts appliqués à la paroi sur une largeur égale à la demi-distance des deux tirants qui l'encadrent, soit : $2,44 \mathrm{~m}$. ( $T_{\text {id }}$ et $T_{\text {ig }}$ étant les tensions des deux tirants de la nappe i étayant le panneau de mesures) on a donc :

$$
T_{i}=\frac{T_{i d}+T_{i g}}{2 \times 2,44}
$$

Les mesures de pression des terres étant relativement dispersées, leurs résultantes ont été calculées à partir de diagrammes moyens. D'autre part, le terme $\frac{d N}{d z}$ a été évalué en affectant la valeur $E=30 \mathrm{GPa}\left(3 \times 10^{10} \mathrm{~Pa}\right)$ au module d'Young de la paroi.

L'analyse des mesures (le tableau 2 relatif à l'étude de l'équilibre en dernière phase est présenté à titre d'exemple) fait apparaitre :

- que pour toutes les phases étudiées, la condition d'équilibre des forces est vérifiée à moins de $12 \%$ près au-dessus des 3 premiers points de moment fléchissant maximum, mais seulement à $21 \%$ près au-dessous du quatrième (Tableau 2).

- qu'entre deux points consécutifs de moment fléchissant maximum, l'écart relatif entre la pression des terres résultante et la composante horizontale de la tension d'ancrage est au maximum $15 \%$ par rapport à cette dernière, audessus du troisième point de moment fléchissant maximum, mais atteint $58 \%$ entre le troisième et le quatrième (Tableau 2).

- que la condition de moment nul, vérifiée à moins de $5 \%$ près pour la plupart des phases de travaux ne l'est plus qu'à $11 \%$ en avant-dernière phase et à $17,5 \%$ près en dernière phase (Tableau 2), c'est-à-dire lorsque sont prises en compte dans le calcul du moment de la pression des terres les valeurs mesurées au-dessous du troisième point de moment fléchissant maximum, qui se situe un peu audessus du toit des marnes et caillasses.

Les mesures de tension d'ancrage étant considérées comme les plus fiables en raison du caractère éprouvé des techniques de mesure mises en œuvre, l'étude de l'équilibre de la paroi semble indiquer que l'erreur par excès affectant la pression des terres mesurée au niveau des alluvions et du sable de Beauchamp est inférieure en moyenne à $10 \%$ des valeurs déterminées expérimentalement mais est 3 à 4 fois plus forte au niveau des marnes et caillasses (tout au moins à la partie inférieure).

Toutefois en raison de l'écart important constaté entre les tensions des tirants de la $4^{\mathrm{e}}$ nappe du panneau de mesures, on ne peut exclure qu'une partie de la pression des terres à la base de ce panneau soit équilibrée par les panneaux voisins (réactions latérales non nulles). Dans 


\begin{tabular}{|c|c|c|c|c|c|c|}
\hline j & $\int_{0}^{z_{m i}} \begin{array}{l}p d z=A \\
(k N)\end{array}$ & $\sum_{1}^{j} T_{i} \cos d_{i}+\frac{e}{2} \frac{d N_{i}}{d z}=B$ & $\frac{A-B}{B}$ & $\int_{z_{m j-1}}^{z_{m i}} \quad \begin{array}{l}(k N) \\
\end{array}$ & $\begin{array}{c}T_{j} \cos d_{j}=D \\
(k N)\end{array}$ & $\frac{C-D}{D}$ \\
\hline 1 & 154 & 152,5 & 1 & & & \\
\hline 2 & 428 & 405,5 & 5,5 & 274 & 253 & 8,3 \\
\hline 3 & 716,5 & 689,5 & 3,9 & 288,5 & 284 & 1,4 \\
\hline 4 & 1228 & 1012 & 21,4 & 512 & 323 & 58,5 \\
\hline & $\int_{0}^{z} p(z-u) d u=E$ & $\begin{array}{l}\sum_{1}^{n} T_{i} \cos d_{i}\left(z-z_{i}\right)=F \\
(m k N)\end{array}$ & $\frac{E-F}{F}$ & & & \\
\hline & 10439 & 8876 & 17,6 & & & \\
\hline
\end{tabular}

Tableau 2 Etude de l'équilibre de la paroi en dernière phase (14/8)

cette éventualité la pression des terres déterminée au niveau des marnes et caillasses pourrait ne pas être affectée d'une erreur systématique supérieure à celles commises au niveau des couches supérieures.

De toutes façons, quelle que soit l'hypothèse retenue, on notera que la pression des terres sur la face arrière reste toujours supérieure à la pression théorique.

\subsection{Moments fléchissants et efforts normaux}

La détermination de ces paramètres à partir des déformations du béton nécessite la connaissance du module d'Young E de la paroi. Celui-ci a été déterminé à partir de l'expression :

$$
\begin{aligned}
\frac{E e^{3}}{12 d} \varepsilon_{M}= & -\int_{0}^{z} p(z-u) d u+\sum_{1}^{n} T_{i} \cos \alpha_{i}\left(z-z_{i}\right) \\
& -e \sum_{1}^{n} T_{i} \sin \alpha_{i}-\frac{E e^{2}}{2} \varepsilon_{M}
\end{aligned}
$$

résultant de la combinaison de (3) (4) et (11).

La résolution de cette équation pour diverses valeurs de z inférieur à $12 \mathrm{~m}$, c'est-à-dire à la profondeur du toit des marnes et caillasses, à partir des données (pression des terres, tensions d'ancrage, valeurs de $\varepsilon_{M}$ et $\varepsilon_{N}$ ) correspondant à différentes phases de travaux conduit aux valeurs moyennes suivantes :

$$
\begin{aligned}
& \mathrm{E}=33,2 \mathrm{GPa} \\
& \mathrm{e}=0,64 \mathrm{~m},
\end{aligned}
$$

la seconde mettant en évidence une surépaisseur de la paroi.

On a alors :

$$
\begin{aligned}
& M(m k N)=3,65 \times 10^{6} \varepsilon_{M} \\
& N(k N)=21,3 \times 10^{6} \varepsilon_{N}
\end{aligned}
$$

L'étude des diagrammes d'effort normal apporte des enseignements intéressants sur le comportement de la paroi. En dépit de l'imprécision du tracé de ces diagrammes sur les 9 mètres supérieurs, il apparaît, qu'à un stade donné des travaux, l'effort normal augmente avec la profondeur jusqu'au niveau du fond de fouille où il atteint sa valeur maximale puis décroit assez rapidement pour se stabiliser au-dessus du pied de paroi (fig. 16). On notera toutefois que des singularités du diagramme d'effort normal apparaissent sur une hauteur d'environ $2.5 \mathrm{~m}$ entre les $3^{\circ}$ et $4^{\circ}$ nappes, lors des deux dernières phases de travaux (fig. 16, $\mathrm{c}$ et d). II semble qu'elles traduisent une fissuration locale du béton de la paroi, auquel cas les relations (15) et (16) ne seraient plus applicables, d'où une indétermination des valeurs de $\mathrm{N}$ et de $\mathrm{M}$ dans cette zone.

L'effort normal en un point de la partie en fiche, étant lié à la composante verticale des forces d'ancrage $\stackrel{n}{\Sigma} T_{i} \sin \alpha_{i}$ 1

et à la résultante $F$ des forces de frottement (comptée positivement vers le bas) au-dessus du point considéré, par l'intermédiaire de la relation

$\mathrm{N}=\mathrm{F}+\sum_{1}^{n} \mathrm{~T}_{i} \sin \alpha_{i e}$

la diminution de $\mathrm{N}$ au-dessous du fond de fouille implique la mobilisation d'un effort de frottement ascendant dans la partie en fiche.

Par ailleurs, le frottement $F_{t}$ du sol sur la face arrière audessus d'un point de la partie hors fiche peut être évalué à partir de la relation (6). D'où l'inclinaison moyenne des contraintes exercées par le sol sur la face arrière au-dessus de ce point:

$\operatorname{tg} \dot{\delta}=\frac{F_{t}}{\int_{0}^{z} p d z}$

L'étude des variations de $\delta$ sur la hauteur au contact des alluvions et du sable de Beauchamp au cours des travaux (Tableau 3) indique une augmentation de ce paramètre jusqu'à ce que la paroi soit dégagée sur environ la moitié de sa hauteur, suivie par une diminution importante au cours des phases ultérieures de travaux.

Le mécanisme de mobilisation du frottement sol-paroi semble donc être le suivant. L'ouverture de la fouille provoque un soulèvement du sol devant la paroi, Celui-ci 
entraîne le développement sur la face avant d'un frottement, qui tend à déplacer la paroi vers le haut. Le sol à l'arrière de la paroi, ainsi que la composante verticale des forces d'ancrage s'opposent à ce mouvement en mobilisant un frottement dirigé en sens inverse. Au-delà d'une certaine profondeur d'excavation la hauteur de contact du sol avec la face avant diminuant, la paroi est moins sollicitée vers le haut d'où une réduction de l'inclinaison des contraintes sur la face arrière.

\begin{tabular}{l|c|c|c}
\hline \multirow{2}{*}{ PHASE } & \multicolumn{2}{|c|}{$\mathrm{F}_{\mathrm{t}} \int_{0}^{z} \mathrm{pdz}$} & tgo \\
& \multicolumn{1}{|c|}{$(\mathrm{kN})$} & \multicolumn{1}{|c}{$(\mathrm{kN})$} & \\
\hline $26 / 6$ & 312,5 & 615 & 0,51 \\
\hline $25 / 7$ & 486 & 747 & 0,65 \\
\hline $8 / 8$ & 163,5 & 753 & 0,22 \\
\hline $14 / 8$ & 141 & 745 & 0,19 \\
\hline
\end{tabular}

Tableau 3 Variations de l'inclinaison moyenne des contraintes exercées par les alluvions et le sable de Beauchamp sur la face arrière de la paroi

Les diagrammes de moment fléchissant n'appellent aucun commentaire particulier, leur allure étant celle attendue pour un écran souple homogène. On remarquera cependant qu'ils n'ont pu être déterminés avec une précision relativement bonne, qu'en raison des efforts de compression importants qui ont empêché la fissuration du béton et ont permis de ce fait d'interpréter les mesures extensométriques à partir des formules de la résistance des matériaux applicables aux poutres homogènes.

\section{Comparaison des mesures et du calcul}

Les résultats expérimentaux obtenus après que le fond de fouille eut atteint sa cote définitive au droit du panneau étudié ont été comparés aux valeurs théoriques, fournies par le calcul aux éléments finis d'une part, et par le calcul en poutre élastique tenant compte d'une réaction élastoplastique du sol (calcul au module de réaction), d'autre part.

\subsection{Méthodes et hypothèses de calcul}

Le calcul aux éléments finis a été effectué en tenant compte d'un comportement purement élastique du sol. Les valeurs du module d'Young du sol ont été déduites des valeurs $E_{0}$ du module tangent à l'origine des courbes contraintes-déformations obtenues à partir des essais triaxiaux consolidés-drainés, le module d'Young affecté à chaque couche étant le module $E_{0}$ correspondant à une étreinte latérale $\sigma_{3}$ égale à la pression verticale du sol à mi-hauteur de la couche considérée. Le coefficient de Poisson du sol a été pris égal à $v=0,3$. Le contour du modèle et les conditions de déplacement aux limites sont représentés figure $20 \mathrm{a}$, sur laquelle ont également été reportées les caractéristiques élastiques attribuées au sol et à la paroi. Le calcul a été effectué au moyen du logiciel ROSALIE (Guellec et al. 1976) en supposant la fouille réalisée en une seule étape.

Le calcul au module de réaction a été effectué en affectant successivement aux différentes couches de sol. 3 ensembles de valeurs du module de réaction $k_{\mathrm{h}}$ (fig. 20b) proportionnelles au module d'Young, les coefficients de proportionnalité étant choisis de facon que les 3 valeurs de $K_{h}$ attribuées à chaque couche couvrent le domaine de variation généralement admis pour ce paramètre compte tenu de la nature du sol (il n'existe actuellement aucune méthode permettant de déterminer la valeur de $K_{h}$ dans la partie hors fiche d'un soutènement). La distribution initiale de la pression du sol sur la paroi -a été définie à partir des valeurs de $K_{0}$ données par la formule de Jaky (fig. 20b), les valeurs limites de la réaction du sol étant déterminées à partir des paramétres de résistance au cisaillement drainé. Les calculs qui tiennent compte des phases intermédiaires de travaux ont été faits au moyen du logiciel établi par S.I.F. Entreprise Bachy (Boudier et al. 1969).

Fig. 20 Hypothèses de calcul :

a) calcul aux éléments finis

b) calcul au module de réaction
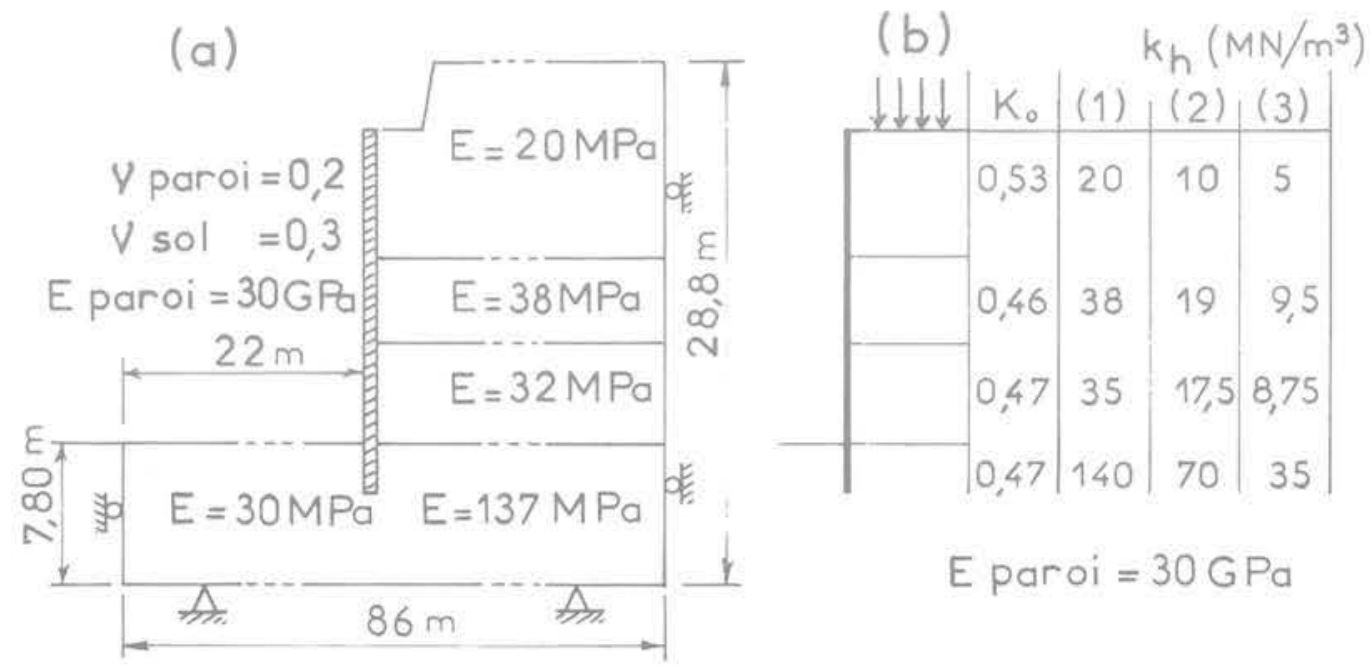
Les tensions de précontrainte introduites dans les deux types de calcul sont les tensions moyennes de chaque couple de tirants d'une même nappe mesurées immédiatement après leur mise en service.

Les principaux résultats de ces calculs (pression des terres, moments fléchissants, déplacements) sont représentés figure 21, ainsi que les valeurs correspondantes établies sur une base expérimentale.

\subsection{Comparaison avec les résultats expérimentaux}

\section{Pression des terres}

Dans la partie hors fiche, y compris à la partie supérieure des marnes et caillasses, les mesures concordent relativement bien avec les valeurs calculées, les premières étant toutefois systématiquement supérieures aux secondes. L'écart entre les mesures et le calcul au module de réaction est sensiblement plus important.

A la partie inférieure de la paroi, les pressions calculées par les éléments finis et les pressions mesurées divergent fortement. Ceci tient, au moins en partie, au fait que le calcul élastique n'impose aucune limite aux contraintes dans le sol et ne peut tenir compte d'une plastification locale au sol, telle que celle qui s'est vraisemblablement produite côté fouille sur le tiers supérieur de la fiche. En revanche. dans la partie hors fiche, où la pression sur la face arrière est toujours supérieure à la pression à l'équilibre limite de poussée, le schéma élastique est bien adapté.

\section{Moments fléchissants}

Compte tenu d'une plastification locale probable du sol en fond de fouille, les diagrammes de moment fléchissant obtenus à la base de la paroi, par le calcul élastoplastique au module de réaction, doivent être considérés comme les plus réalistes du moins en ce qui concerne leur allure, car, contrairement à une opinion très répandue, les valeurs du moment fléchissant sont très sensibles aux valeurs du module de réaction. Dans la moitié inférieure de la paroi, le diagramme expérimental se situe dans la zone délimitée par les diagrammes théoriques obtenus à partir des valeurs extrêmes du module de réaction. Dans la partie supérieure, les divergences entre le diagramme expérimental et les diagrammes obtenus par les deux méthodes de calcul sont très importantes, le calcul aux éléments finis conduisant toutefois aux valeurs les moins discordantes.

\section{Déplacements}

Le calcul aux éléments finis donne un ordre de grandeur assez satisfaisant des déplacements de la tête de paroi ( $30 \mathrm{~mm}$ pour une valeur réelle d'au moins $45 \mathrm{~mm}$ ) et du point de scellement du tassomètre $(19 \mathrm{~mm}$ pour une valeur réelle d'au moins $30 \mathrm{~mm}$ ). II met également en évidence l'importance de la zone de décompression du sol à l'arrière de la paroi, le calcul indiquant un déplacement horizontal de la surface du sol égal à $5 \mathrm{~mm}$ à 60 mètres de la tête de paroi, soit à une distance de celle-ci correspondant à trois fois la profondeur totale de la fouille.

En revanche le calcul au module de réaction conduit à des déplacements très inférieurs aux déplacements réels. Dans le cas étudié, cette sous-estimation des déplacements résulte essentiellement du fait que ce type de calcul ne tient pas compte des déplacements du sol en arrière des points de scellement théoriques des tirants, ceux-ci étant considérés comme fixes.

\section{Tensions d'ancrage}

La comparaison des tensions moyennes mesurées pour chaque couple de tirants avec les valeurs calculées (tableau 4) ne fait pas apparaittre de divergences très importantes entre les mesures et le calcul. Le calcul aux

Fig. 21 Comparaison des résultats du calcul avec les résultats expérimentaux obtenus en dernière phase de travaux : a) pression des terres

b) déplacements

c) moments fléchissants

Les surfaces hachurées représentent le domaine de variation des résultats du calcul au module de réaction compte tenu des valeurs extrêmes admises pour $K_{H}$

PRESSION DES TERRES $\left(K P_{a}\right)$

DEPLACEMENT(mm) MOMENTS FLECHISSANTS ( $\mathrm{kN}$ )

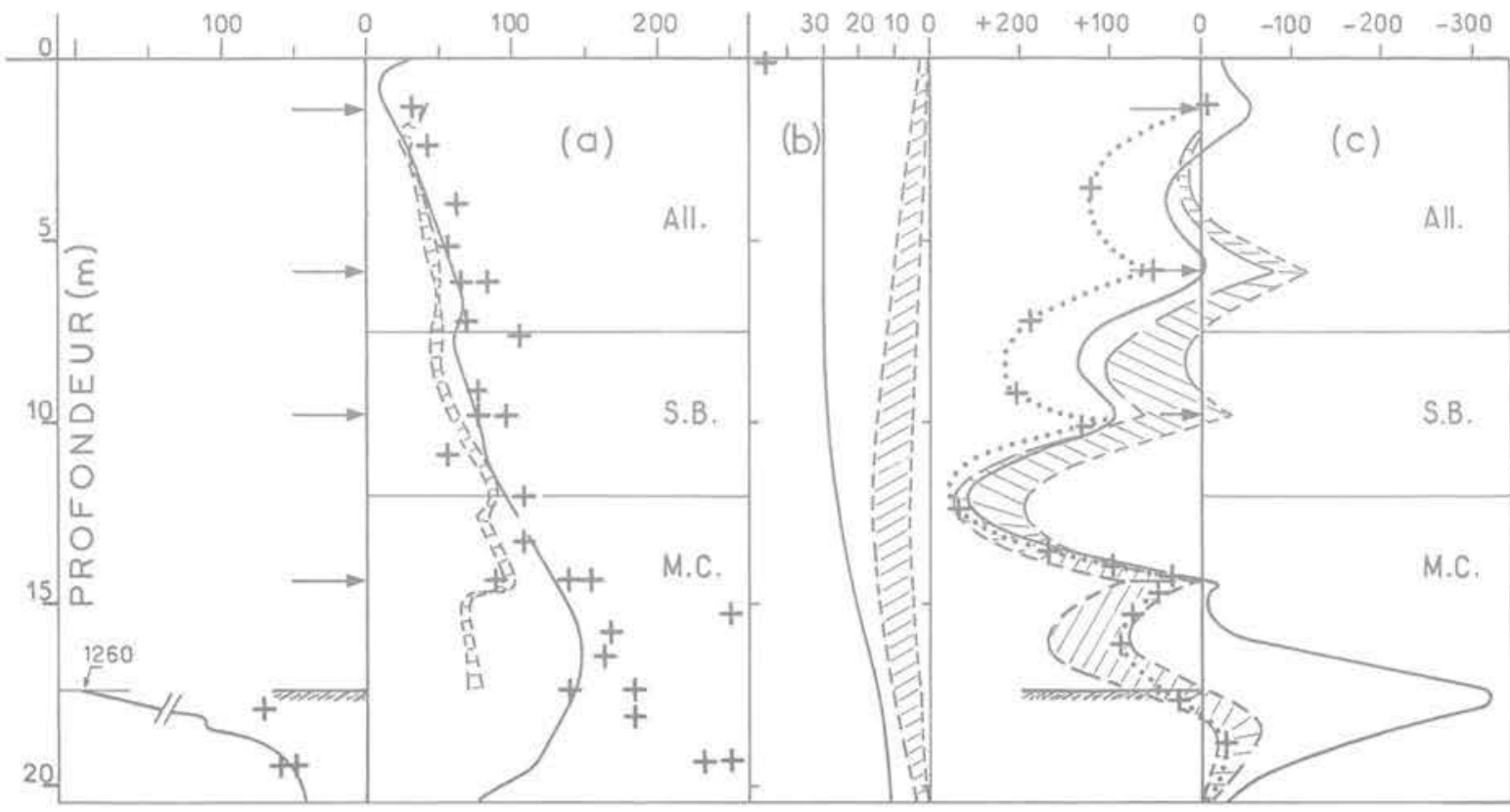


éléments finis donne toutefois des résultats plus satisfaisants sauf en ce qui concerne la $4^{e}$ nappe.

\begin{tabular}{c|c|c|c|c|c}
\hline \multicolumn{2}{c|}{ Nappe } & 1 & 2 & 3 & 4 \\
\hline $\begin{array}{c}\text { Tension effective de } \\
\text { précontrainte (kN) }\end{array}$ & 324 & 635 & 704 & 845 \\
\hline \multirow{4}{*}{$\begin{array}{c}\text { Tension } \\
\text { finale }\end{array}$} & $\begin{array}{c}\text { méléments } \\
\text { finis }\end{array}$ & 359 & 691 & 804 & 1010 \\
\cline { 2 - 6 } & $\begin{array}{c}\text { mesuré } \\
\text { calcul en } \\
\text { (kN) }\end{array}$ & & 700 & 770 & 870 \\
\cline { 2 - 6 } & poutre & 329 & 643 & 725 & 855 \\
\hline
\end{tabular}

Tableau 4 Comparaison des tensions d'ancrage mesurées et calculées en phase finale

II apparaît donc que les résultats du calcul aux éléments finis concordent mieux, dans l'ensemble, avec les résultats expérimentaux que ceux obtenus par le calcul au module de réaction. Ceci résulte en particulier du fait que le schéma de comportement du sol considéré dans le premier type de calcul est relativement bien adapté au cas étudié, alors que le second ne peut tenir compte des déplacements des points de scellement des tirants, qui semblent jouer un rôle assez important vis-à-vis du comportement de l'ouvrage.

\section{Conclusions}

L'étude expérimentale de la paroi moulée de la S.E.R.E.T.E. apporte un certain nombre d'informations relatives aux appareillages et techniques de mesure utilisés, au comportement de la paroi et à la validité des méthodes de calcul employées.

Les appareillages permettant d'obtenir les valeurs des tensions d'ancrage (cales dynamométriques), les déformées relatives de la paroi (inclinomètre) et les déformations relatives de béton (extensomètres à corde vibrante) se sont, dans l'ensemble, avérés fiables et suffisamment sensibles, les difficultés rencontrées au cours de l'interprétation étant liées essentiellement à un nombre insuffisant d'extensomètres à la partie supérieure de la paroi et à l'interruption momentanée des mesures inclinométriques. Les résultats fournis par les capteurs de pression totale utilisés pour la mesure de la pression des terres sont sensiblement moins précis, vraisemblablement en raison des conditions de contact sol-capteur qui peuvent être très différentes pour des capteurs intéressant une même couche. On notera cependant que la dispersion des mesures n'est pas seulement due aux conditions de fonctionnement des capteurs, mais reflète certainement une distribution irrégulière de la pression sur la paroi due à l'hétérogénéité du sol et au vrillage du panneau de mesures résultant des conditions particulières d'exécution des terrassements. Quoi qu'il en soit, le nombre important de capteurs implantés sur la face arrière de la paroi a permis d'obtenir un ordre de grandeur acceptable de la pression du sol sur cette face.

En ce qui concerne le comportement de la paroi, l'étude expérimentale a permis de déterminer, aux divers stades des travaux, la distribution de la pression des terres sur la face arrière de la paroi, la distribution des moments fléchissants et des efforts normaux ainsi que les valeurs des tensions d'ancrage. Elle a plus particulièrement montré :

- que la pression initiale des terres sur la paroi n'est vraisemblablement pas en relation simple avec le coefficient de pression du sol au repos:

- que le comportement du sol à l'arrière de la paroi est un comportement « élastique », la pression sur la face arrière restant supérieure à celle correspondant à l'équilibre limite de poussée :

- que les déplacements de la paroi vers la fouille, qui atteignent en tête de paroi une amplitude supérieure à $2 / 1000$ de sa hauteur totale, résultent de la décompression du sol dans une zone très étendue à l'arrière de l'ouvrage. Cette décompression est, en effet, encore sensible à une distance de la paroi supérieure à trois fois la profondeur de la fouille :

- que l'importance des efforts de compression axiale appliqués à la paroi (dus à la composante verticale des forces d'ancrage et au frottement sol-paroi) a empêché la fissuration du béton et que de ce fait la paroi s'est comportée comme un écran souple homogène.

Cette étude a en outre permis de mettre en évidence le mécanisme de la mobilisation du frottement sol-paroi.

La confrontation des mesures aux résultats du calcul montre que le calcul aux éléments finis, bien que basé sur des schémas très simplifiés (comportement purement élastique du sol, phases intermédiaires de travaux non prises en compte), donne une image assez réaliste du comportement de l'ouvrage et du sol qui l'environne.

Les résultats du calcul au module de réaction concordent moins bien avec ceux déduits des mesures, principalement en ce qui concerne les déplacements de la paroi dont les valeurs théoriques sont très inférieures aux valeurs réelles. Ces divergences entre les mesures et le calcul au module de réaction sont dues essentiellement au fait que celui-ci ne tient pas compte des mouvements du sol qui se produisent à l'arrière de la paroi, à l'extérieur de la zone intéressée par les tirants, mouvements qui, dans le cas étudié, semblent avoir une influence assez sensible sur le comportement de l'ouvrage.

Les auteurs tiennent à exprimer leur gratitude

à MM. Deroy et Thomann, Ingénieurs au L.C.P.C., qui ont effectué les calculs aux éléments finis de la paroi.

à M. Makhoul, ex-stagiaire au L.C.P.C., pour sa collaboration à l'analyse des mesures,

à M. Luong, Ingénieur au Laboratoire de Mécanique des Solides de l'École Polytechnique,

pour les discussions fructueuses qu'ils ont eues avec lui. au cours de cette étude.

\section{Références bibliographiques}

BOUDIER J GILLARD J MASTIKIAN L (1969) - Calcul de stabilité des parois moulées sur ordinateur - Comparaison avec des observations faites in situ - Cas particulier d'une enceinte cylindrique \#, Compte rendu du $5^{\mathrm{e}}$ Congrès International de Mécanique des Sols et des Travaux de Fondation, Mexico, Sessions Spéciales 14 et 15 , p. 45-49.

DELMAS F., GANDAIS M. HABIB P., JOSSEAUME H., LUONG M-P., PILOT G. (1977), - "Comportement d'un soutènement en paroi moulée ע. Compte rendu du $9^{\circ}$ Congrès International de Mécanique des Sols et des Travaux de Fondation. Tokyo Vol. 2 , p. 43-46, Bull. de liaison des Laboratoires des Ponts et Chaussées, Numéro spécial VI F, p. 70-73.

DEROY J.-M. (1974). - "Settlement behind anchored walls \#, Master of Sciences Thesis, University of Alberta.

GUELLEC P., HUMBERT P. RICARD A. (1976), - " La méthode des éléments finis et le système Rosalie y, Bull. de liaison des Laboratoires des Ponts et Chaussées, $n^{\circ} 81$, p. 152-162. 